\title{
Cerebrovascular Diseases
}

Abbott, A.L. 362

Abboud, H. 216

Adams, H. 75

Ahn, S.H. 289

Ajdacic-Gross, V. 211

Akagawa, H. 46

Alecu, C. 441

Alexandrov, A.V. 256, 325

Al-Shahi, R. 318

Amarenco, P. 75, 216

Anderson, C.S. 162

Arboix, A. 353

Arnold, M. 318

Auch, D. 140

Auckland Regional Community Stroke Study Group 162

Bachmann, R. 275

Bagher-Ebadian, H. 91

Barber, M. 260

Barber, P.A. 162

Barlow, N. 260

Baron, J.-C. 35

Bartrés-Faz, D. 353

Bäzner, H. 243

Begachev, A.V. 50

Bejot, Y. 126

Benatru, I. 126

Benessiano, J. 216

Benlloch, V. 6

Benslamia, L. 299

Bereczki, D. 388

Bertrand, M. 299

Bevan, S. 148

Bhatia, R. 456

Biasi, V. 20

Blanco, M. 85

Bogousslavsky, J. 466

Bokeriia, L.A. 50

Bonita, R. 162

Bonora, E. 20

Boode, B. 294

Bornstein, N. 75

Bousser, M.G. 299

Bravo, Y. 85

Breskina, N.Y. 50

Busschbach, J.J.V. 401

Caillier, M. 126

Cakmak, S. 462

Çallı, C. 331

Canbaz, D.H. 331

Carpén, O. 27
Supplement issues have their own Author Index
Carter, K.N. 162

Castellanos, M. 85

Çelebisoy, M. 331

Çelebisoy, N. 331

Chamarro, R. 6

Chambers, B.R. 362

Chan, D.K.Y. 181

Chan, K.H. 69

Chen, L. 381

Cheng, C.-A. 309

Chengodu, T. 424

Cheung, R.T.F. 69

Chhabra, J. 304

Chiu, S.-W. 309

Cho, A-H. 14

Choi, H.-Y. 289

Choi, J. 103

Choi-Kwon, S. 103

Chopp, M. 91

Christensen, M.C. 57, 221

Chung, E.M.L. 169

Claeys, I. 339

Cocho, D. 85

Cordato, D. 181

Cordonnier, C. 318

Cottin, Y. 126

Couvreur, G. 126

Csiba, L. 75, 388

Daly, K. 424

Darlington, A.-S.E. 401

Davydov, D.M. 50

de Bray, J.M. 441, 448

de Haan, E.H.F. 408

de Kort, P.L.M. 408

De Reuck, J. 339

Dehoux, M. 216

Dempsey, E. 66

Derendorf, H. 72

Derex, L. 462

Desvarieux, M. 75

Deuschl, G. 188

Díaz-Manera, J. 85

Dichgans, M. 318

Diener, H.-C. 368

Dippel, D.W.J. 401, 435

Dittrich, R. 275

Donnan, G.A. 362

Dubas, F. 448

Ducrocq, X. 441

Durier, J. 126

Dziedzic, T. 395
Ebrahim, S. 75

Eng, J.J. 203

Engelter, S.T. 211

European Stroke Initiative executive committee 344

Evans, D.H. 169

Ewing, J.R. 91

Falvo, N. 126

Fan, L. 169

Farrell, M.A. 66

Fatar, M. 75

Figlewicz, D.A. 395

Flegar-Meštrić, Z. 430

Foley, N. 194

Forteza, A. 456

Fortrat, J.O. 441

Franke, C. 294

Fromont, A. 126

Fukui, D. 109

Gass, A. 4

Gennaro, N. 20

Gerigk, L. 175

Gesztelyi, R. 388

Ghandehari, K. 132

Ghofrani, H.A. 140

Giroud, M. 126

Golukhova, E.Z. 50

Gormley, K. 148

Gostynski, M. 211

Grau-Olivares, M. 353

Grga, A. 430

Grieco, G. 73

Grimminger, F. 140

Groden, C. 175

Guillon, B. 299

Habbema, J.D.F. 435

Hacke, W. 344, 465

Hackett, M.L. 162

Halloul, Z. 458

Han, S.W. 289

Hayakawa, M. 381

Hebrang, A. 430

Hecht, M. 140

Heidbreder, A. 275

Heinemans, C.T.I. 40

Hennerici, M.G. 1, 75, 243, 256

Heo, J.H. 289

Hernandez Hernandez, R. 75

Heuschmann, P. 275

Hori, T. 46
Horn, P. 175

Hsu, Y.-D. 309

Hung, W.T. 181

Ibayashi, S. 109

Ijäs, P. 27

Inoue, I. 46

Inoue, T. 109

Irie, K. 381

Izadi, Z. 132

Jaff, M. 75

James, M.A. 156

Jansen, B.P.W. 408

Januš, D. 430

Jeon, S.-B. 459

Jiang, Q. 91

Johnson, P. 156

Jonas, S. 73

Joshi, A.V. 57

Junqué, C. 353

Kajiyama, K. 35

Kang, D.-W. 14, 103, 459

Kanno, T. 381

Kapke, A. 91

Kappelle, L.J. 408

Kaps, M. 140

Karagiozov, K.L. 381

Kaste, M. 27, 344, 465

Kasuya, H. 46

Kataoka, H. 315

Kato, Y. 381

Kazanovskaya, S.N. 50

Kéki, S. 388

Kelly, P.J. 66

Kemmling, A. 175

Kim, J.S. 14, 103, 459

Kimura, K. 282

Kjellström, T. 229

Klimkowicz-Mrowiec, A. 395

Koch, S.. 456

Köhrmann, M. 83

Kopec, G. 395

Koudstaal, P.J. 435

Kovanen, P.T. 27

Kownator, S. 75

Kreisel, S.H. 243

Krischek, B. 46

Kuhlenbäumer, G. 275

Kumral, E. 331

Kwakkel, G. 40

Kwon, S.U. 14, 103, 459 
Labreuche, J. 216

Láinez, M.J.A. 6

Langhorne, P. 320, 464

Lao, A.Y. 325

Larson III, T.C. 453

Lavallee, P.C. 216

Lee, D.K. 459

Lee, J.-T. 309

Lee, J.Y. 289

Lee, N.S. 304

Lee, R. 69

Lee, W.C. 57

Lenzi, G.L. 121

Lepäntalo, M. 27

Leung, G.K.K. 69

Levi, C.R. 362

Leys, D. 344, 465

Lhoste, P. 448

Lin, J.-C. 309

Lin, W.-W. 309

Lindeman, E. 40

Lindsberg, P.J. 27

Liu, X. 117

Liu-Ambrose, T. 203

López-Mut, J.V. 6

Lu, M. 91

Lui, W.M. 69

Lynch, T. 66

Lyrer, P.A. 211

Maasland, L. 435

Macfarlane, P.W. 260

Maestre, J. 85

Magyar, M.T. 388

Makihara, N. 109

Malecki, M. 395

Mali, W.P.T.M. 267

Marc, G. 448

Marie, C. 126

Markus, H.S. 148, 323

Martí-Fàbregas, J. 85

Martí-Vilalta, J.-L. 85

Mast, H. 318

Mäyränpää, M.I. 27

Mazighi, M. 216

McCollum, C. 424

McCullough, L.D. 304

McGuire, A.J. 221

McHugh, J.C. 66

Meairs, S. 75, 319

Meseguer, E. 216

Michallet, M. 462

Millán, M. 85

Millerot, E. 126

Minematsu, K. 109, 282

Mitsias, P.D. 91

Miyashita, F. 282

Moreau, T. 126

Morton, J.J. 260

Muggeo, M. 20
Nabavi, D.G. 275

Nakajima, M. 282

Nam, H.S. 289

Narita, A. 46

Naritomi, H. 282

Nassenstein, I. 275

Navarro, J.C. 325

Naylor, A.R. 169

Nedeltchev, K. 318

Negoro, M. 381

Nighoghossian, N. 462

Norrving, B. 229

Nuotio, K. 27

Nys, G.M.S. 408

Obach, V. 85

Okada, Y. 109

Olivot, J.-M. 216

Osseby, G.-V. 126

Pang, M.Y.C. 203

Papa, S. 211

Park, I.C. 289

Participants of the European Stroke Workshop 320

Pasco, A. 448

Pascual, A.M. 6

Pashos, C.L. 57

Passchier, J. 401

Patel, S.C. 91

Pautot, V. 448

Peng, G.-S. 309

Pera, J. 395

Perkov, S. 430

Petersen, N.H. 188

Peynet, J. 299

Plendl, H. 188

Polunina, A.G. 50

Prati, P. 75

Preden-Kereković, V. 430

Puig, M. 85

Raikou, M. 221

Ribbers, G.M. 401

Ringelstein, E.B. 275, 344, 465

Rinkel, G.J.E. 318

Roditi, G. 260

Rohsbach, D. 275

Rønning, O.M. 417

Roquer, J. 85

Rosengarten, B. 140

Rosewell, M. 156

Rouaud, O. 126

Rovira, M. 353

Rundek, T. 75

Ryan, A.M. 66

Sacco, R. 368

Sadato, A. 381

Saimanen, E. 27
Sairanen, T. 27

Saksi, J. 27

Salonen, O. 27

Salter, K. 194

San Román, L. 85

Sano, H. 381

Sastry, S. 424

Scharf, J. 175

Schellinger, P.D. 83

Schepers, V.P.M. 40

Schermuly, R.T. 140

Schmied, A.B. 188

Schmiedek, P. 175

Schminke, U. 75

Schoof, J. 458

Sharma, V.K. 325

Shatchkute, A. 229

Shen, Q. 181

Sheng, A.Z. 181

Shimode, A. 282

Silverman, I.E. 304

Simon, O. 216

Sitzer, M. 75

Skalej, M. 458

Slowik, A. 395

Soinne, L. 27

Soler, J. 6

Soliva, J.-C. 353

Soltanian-Zadeh, H. 91

Stack, J. 66

Stapf, C. 318

Stavem, K. 417

Steering Committee and PRoFESS Study Group 368

Steg, P.G. 216

Steiner, T. 318

Stork, J.L. 362

Stott, D.J. 260, 320

Szczudlik, A. 395

Szermer, P. 395

Tajima, A. 46

Takasawa, M. 35

Tanizawa, E. 315

Tardif, J.-C. 75

Targa, C. 353

Taylor, A. 75

Teasell, R. 194

Thomas, B.P. 453

Török, J. 388

Touboul, P.-J. 75

Toyoda, K. 109

Tsivgoulis, G. 325

Turaj, W. 395

Tzourio, C. 299

Uchino, M. 282

Ueno, S. 315

Uwatoko, T. 109
Valikovics, A. 388

van Balen, R. 401

van de Port, I.G.L. 40

van der Worp, H.B. 267, 318

van Leeuwen, M.S. 267

Van Maele, G. 339

van Oostenbrugge, R. 294

van Zandvoort, M.J.E. 408

Vanhee, F. 339

Velthuis, B.K. 267

Venturi, C. 20

Verhagen, H.J.M. 267

Verlato, G. 20

Vespignani, H. 441

Vicaut, E. 75

Vicenzini, E. 121

Vidjak, V. 430

Vielle, B. 448

Vrhovski-Hebrang, D. 430

Waaijer, A. 267

Wahlgren, N. 319

Watanabe, S. 381

Welzen, V. 294

Whittle, I. 221

Wloch, D. 395

Wolkow, P. 395

Woo, K.S. 75

Wright, F. 320

Wu, W. 117

Wunderlich, M.T. 458

Xu, G. 117

Yamaguchi, T. 35

Yamamoto, S. 35

Yasumori, K. 109

Yin, Q. 117

Yin, S.-J. 309

Yoneda, M. 381

Yusuf, S. 368

Zamboni, C. 20

Zannad, F. 75

Zeevi, N. 304

Zeller, J.A. 188

Zeller, M. 126

Zhang, R. 117

Zhang, Y.Y. 181

Zoppini, G. 20

Zsuga, J. 388

Zsuga, M. 388

Zunker, P. 188

Zureik, M. 75

Zyss, J. 462 